\title{
Guidelines, Pathways, Bundles, and Protocols: Clinical Recipes for Success
}

The development and implementation of clinical practice guidelines have been shown to improve patient care and outcomes and have been well described for over 2 decades. Although there are a myriad of terms such as pathways, protocols, care maps, and bundles, the fundamental aim is the same: evidence from clinical trials and associated research informs clinicians of best approaches for the delivery of care, prescription of medications, and application of technology. ${ }^{1}$ The development of clinical practice guidelines is a tremendous undertaking and typically entails the convening of a multidisciplinary group, usually composed of subject matter experts who agree to collaborate to achieve a common goal while concentrating on improved quality, safety, and outcomes for a particular patient population and increasingly with economic consideration. ${ }^{1}$ The development of a national approach to surveillance for ventilator-associated events, sponsored by the Centers for Disease Control and Prevention, serves as a good example. ${ }^{2}$

In their summary of a previously published Cochrane review, Rotter et $\mathrm{al}^{3}$ described the merits of clinical pathways, the effects on professional practice and patient outcomes, and economic impact. Data from studies had to meet four criteria to be considered in the final analysis, which included 27 studies from over 3,000 that were evaluated. Characteristics of the selected studies included primarily randomized controlled trials in 8 countries, with the majority comparing stand-alone clinical pathways with traditional practice. Their findings support the creation of clinical pathways, as the results demonstrated improved patient safety, cost-effective resource allocation, and efficiency of care and indicated that costs and stay could be reduced. The authors suggested that a more universal approach to clinical pathway definition and design needs to be established for improved comparisons.

In this issue of Respiratory CARE, Mola et $\mathrm{al}^{4}$ present their work with the use of a respiratory care bundle aimed

\footnotetext{
Mr Betit has disclosed no conflicts of interest.
}

Correspondence: Peter Betit RRT-NPS FAARC, Department of Respiratory Care, Boston Children's Hospital, MA-883, 300 Longwood Avenue, Boston, MA 02115. E-mail: peter.betit@childrens.harvard.edu.

DOI: $10.4187 /$ respcare.03985 at improving the quality of care in very low birthweight infants and reducing the incidence of bronchopulmonary dysplasia (BPD). They sought to evaluate the sustainability of this quality improvement initiative across four $2-y$ time periods spanning 2002 to 2010 . The bundle is a compilation of evidence-based practices, including early surfactant replacement therapy, postextubation CPAP, and

\section{See the Original Study on Page 309}

targeted $\mathrm{S}_{\mathrm{pO}_{2}}$ parameters, and agreed-upon best practices, including standardized supplemental oxygen administration and a respiratory therapist-driven ventilator management protocol. The principle aim of the bundle was to reduce the incidence of BPD, which was demonstrated in the first 3 eras but not in the most recent epoch.

Implementation of the bundle had been successful with respect to the introduction and consistent application of CPAP and a reduction in exposure to high levels of oxygen, with a subsequent decrease in invasive ventilator days. However, in the most recent time period, there was a notable increase in BPD and a decrease in survival without $\mathrm{BPD}$, triggering a debate as to the bundle's lack of sustainability, for which the authors pose a number of potential contributing factors. One such factor was the limited availability of T-piece resuscitators and blended oxygen capabilities; another was the unknown frequency with which caregivers utilized manual ventilation to manage acute decompensation events such as unplanned extubations. Manual ventilation events and techniques were not tracked and could have contributed to lung injury. The authors further acknowledged the limitations of a retrospective review over such a long duration.

It may be unreasonable to expect a guideline that was developed in 2002 to be as effective in achieving its goals several years later. For instance, although an attempt was made to bring uniformity to ventilator management, there may have been unrealized nuances in approach such as timing of ventilator changes and a commitment to strict adherence to the bundle, which could have contributed to the findings of the study. A research protocol typically has well defined parameters and clear end points developed to answer a specific question, whereas a quality improvement project is ongoing and may be subject to unintended vari- 
ations as therapies, medications, and technology evolve, and as participants and stakeholders vary over time.

Rathod et $\mathrm{al}^{5}$ have proposed a new approach to clinical practice guideline development and evaluation through the coalescence of business management theories and evidencebased medicine into a Standardized Clinical Assessment and Management Plan (SCAMP). The aim of this quality improvement initiative is to minimize care delivery variations through standardization, improve patient care, assess intervention effectiveness, and reduce unnecessary resource use. The foundation of a SCAMP includes a wellresearched background position paper, a statement of conceivable hypotheses, consensus of experts, and collection of key and specific data. The authors assert that SCAMPs differ from more traditional formats in that ongoing data analyses inform users of beneficial strategies for subgroups and that correlations and trends emerge that will further guide evolving clinical practice.

As the Affordable Care Act gains further traction, it is highly probable that providers will be required to follow established and proven clinical practice guidelines and develop mechanisms for evaluating effectiveness both clinically and economically. The respiratory care bundle evaluated by Mola et $\mathrm{al}^{4}$ may not have had the expected and sustained effect on BPD, but significant strides were made early on in its implementation, and the effort to create an evidence-informed guideline and subsequent appraisal should be lauded. It is possible that a more frequent review of data and factors such as adherence to the bundle, training of newcomers to the process, and appreciation of the subtle changes that occurred over the 8-y span may have had an impact on the occurrence of BPD. The bundle for the care of very low birthweight infants is not necessarily defective, but some of its elements may need revision and updating, and the approach needs to ensure a more iterative process that permits fine-tuning.

Peter Betit RRT-NPS FAARC
Department of Respiratory Care
Boston Children's Hospital
and
Department of Anesthesia
Harvard Medical School
Boston, Massachusetts

\section{REFERENCES}

1. Steinbrook R. Guidance for guidelines. N Engl J Med 2007;356(4): 331-333.

2. Magill SS, Klompus M, Balk R, Burns SM, Deutschman CS, Diekema $\mathrm{D}$, et al. Developing a new, national approach to surveillance for ventilator-associated events: executive summary. Respir Care 2013; 58(11):1985-1989.

3. Rotter T, Kinsman L, James E, Machotta A, Willis J, Snow P, Kugler $\mathrm{J}$. The effects of clinical pathways on professional practice, patient outcomes, length of stay, and hospital costs: Cochrane review and meta-analysis. Eval Health Prof 2012;35(1):3-27.

4. Mola SJ, Annibale DJ, Wagner CL, Hulsey TC, Taylor SN. NICU bedside caregivers sustain process improvement in respiratory care and decrease incidence of bronchopulmonary dysplasia in infants $<30$ weeks gestation. Respir Care 2015;60(3):309-320.

5. Rathod RH, Farias M, Friedman KG, Graham D, Fulton DR, Newburger $\mathrm{J}$, et al. A novel approach to gathering and acting on relevant clinical information: SCAMPs. Congenit Heart Dis 2010;5(4):343353. 